Open Access

\title{
Droxinostat sensitizes human colon cancer cells to apoptotic cell death via induction of oxidative stress
}

Ying Huang ${ }^{1,2+}$, Wuping Yang ${ }^{1,2 \dagger}$, Huihong Zeng ${ }^{2 \dagger}$, Chuan $\mathrm{Hu}^{2}$, Yaqiong Zhang ${ }^{2}$, Nanhua Ding ${ }^{2}$, Guangqin Fan ${ }^{1,3}$, Lijian Shao ${ }^{1,3^{*}}$ and Bohai Kuang ${ }^{2^{*}}$ (i)

* Correspondence: Ishao@ncu.edu. cn; kuangbohai@163.com

†Ying Huang, Wuping Yang and Huihong Zeng contributed equally to this work.

${ }^{1}$ Jiangxi provincial key laboratory of preventive medicine, Nanchang University, Nanchang 330006, China ${ }^{2}$ Medical School of Nanchang University, 461 Bayi Road, Nanchang 330006, Jiangxi, China

Full list of author information is available at the end of the article

\begin{abstract}
Upregulation of histone acetylation plays a critical role in the dysregulation of transcription. It alters the structure of chromatin, which leads to the onset of cancer. Histone deacetylase inhibitors may therefore be a promising way to limit cancer progression. In this study, we examined the effects of droxinostat on the growth of HT-29 colon cancer cells. Our results show that droxinostat effectively inhibited cell growth and colony-forming ability by inducing cellular apoptosis and ROS production in HT-29 cells. Notably, the apoptotic inhibitor Z-VAD-FMK significantly decreased the levels of cellular apoptosis and the antioxidant $y$-tocotrienol (GT3) significantly decreased ROS production induced by droxinostat treatment. Z-VADFMK and GT3 also partially reversed the negative growth effects of droxinstat on HT29 cells. GT3 treatment decreased cellular apoptosis and increased colony-forming ability upon droxinostat administration. Z-VAD-FMK treatment also partially decreased droxinostat-induced ROS production. Our findings suggest that the effects of droxinostat on colon cancer cells are mediated by the induction of oxidative stress and apoptotic cell death.
\end{abstract}

Keywords: Droxinostat, HT-29 cells, Apoptosis, ROS

\section{Introduction}

Colorectal cancer (CRC) is one of the most common malignant tumors of the digestive tract: it is the third most commonly diagnosed cancer and the fourth most common cause of cancer death worldwide [1, 2]. Chemotherapy regimens based on 5-fluorouracil (5-FU) remain the standard treatment for CRC in both adjuvant and advanced disease settings and improves overall survival [3]. However, response rates to 5-FU therapy are between 10 and $20 \%$ in the metastatic setting [4]. Resistance to chemotherapy is still a major reason for treatment failure in colon cancer [5]. Thus, novel and efficacious therapeutic agents and strategies are urgently needed for the treatment of colon cancer.

Histone deacetylase inhibitors (HDACIs) were recently identified as a promising new target in cancer therapy. Multiple studies have demonstrated that HDACIs can arrest cell growth, block angiogenesis, and induce differentiation and apoptosis in tumor cells [6]. Histones are typically catalyzed by two enzyme families: histone acetyltransferases

(c) The Author(s). 2018 Open Access This article is distributed under the terms of the Creative Commons Attribution 4.0 International License (http://creativecommons.org/licenses/by/4.0/), which permits unrestricted use, distribution, and reproduction in any medium, provided you give appropriate credit to the original author(s) and the source, provide a link to the Creative Commons license, and indicate if changes were made. The Creative Commons Public Domain Dedication waiver (http://creativecommons.org/ publicdomain/zero/1.0/) applies to the data made available in this article, unless otherwise stated. 
(HATs) and histone deacetylases (HDACs). Histone acetylation and deacetylation of lysine residues play an important role in the transcriptional regulation of eukaryotic cells $[7,8]$. Subsequent functional inactivation and aberrant gene expression of HAT activity or dysregulation of HDAC activity is reported to contribute to cancer initiation and mediate tumor cell proliferation.

HDACIs are therefore now considered attractive as anticancer drugs. Many HDACIs have been shown to sensitize cells to Fas-mediated apoptosis [9] and several HDACIs can synergize with tumor necrosis factor-related apoptosis-inducing ligand (TRAIL) in many kinds of human cancer but not in normal cells [10]. However, the mechanisms of these interactions may be vary by tumor type and drug, with some requiring deeper investigation. For example, the molecular mechanisms underlying the enhancement of colon cell apoptosis by HDACIs remain elusive.

HDACI-induced apoptosis is one essential part of limiting cancer growth and metastasis [11]. There are two major apoptotic pathways: the extrinsic death receptor-mediated pathway and the intrinsic mitochondria-mediated pathway. The truncated Bid protein accounts for the cross-talk between the two [12, 13]. Activation of the extrinsic death receptor-mediated apoptotic pathway, which involves TRAIL and its corresponding cell surface death receptors, leads to the formation of the death-inducing signaling complex (DISC). This event is followed by the activation of caspase-8 [14-16]. Cellular FADD-like IL-1 $\beta$-converting enzyme-inhibitory protein (c-FLIP) is an important component of DISC with the ability to inhibit the activation of caspase $8[17,18]$. The mitochondria-mediated pathway is characterized by the loss of mitochondrial membrane potential and the release of cytochrome c from the mitochondria into the cytoplasm. This can be initiated by Bcl-2 family proteins [19].

It has been reported that HDAC inhibitors, such as droxinostat, vorinostat and tubastatin A, induce cellular apoptosis by decreasing the expression of c-FLIP and the anti-apoptotic gene Bcl-2, and increasing the expression of pro-apoptotic genes, such as Bax.

Droxinostat, developed by Reed et al. [20-22], inhibits the activities of HDAC3, HDAC6 and HDAC8 and displays promising anticancer activity [23]. Recent studies have demonstrated that droxinostat can sensitize cells of several cancer cell lines, including PC-3, DU-145, OVCAR-3, HepG2 and SMMC-7721, to death receptor ligands [22]. Droxinostat-induced cell death is mediated by the induction of cellular apoptosis, including caspase 8-dependant extrinsic apoptosis [23, 24]. On the other hand, several reports have demonstrated that some HDACIs stress tissues and cells by causing overproduction of reactive oxygen species (ROS) to induce cell death [25]. Cancer cells are very sensitive to oxidative stress [26]. Whether droxinostat induces oxidative stress in cancer cells is unknown.

In this study, we re-investigated whether treatment with HDACIs, such as droxinostat, would inhibit the growth of HT-29 human colon cancer cells. Mechanistically, we not only elucidated the apoptotic effects of droxinostat on HT-29 cells but also the overproduction of ROS induced by droxinostat treatment.

\section{Materials and methods Reagents}

Droxinostat, tubastatin A and PCI-34051 were purchased from ApexBio. FITC-conjugated Annexin V Apoptosis Detection Kit I was purchased from BD Biosciences. Propidium 
iodide (PI) was purchased from Sigma-Aldrich. DCFDA was purchased from Life Technologies. Z-VAD-FMK was purchased from Selleckchem. $\gamma$-tocotrienol (GT3) was purchased from Sigma-Aldrich.

\section{Cell lines and cell culture}

The human colon cancer cell lines used in this study were purchased from the American Type Culture Collection (ATCC). HT-29 or HCT-116 cells were cultured in RPMI medium 1640 supplemented with 10\% heat-inactivated fetal bovine serum (FBS), $5958 \mathrm{mg} / \mathrm{l} \mathrm{HEPES}, 100 \mathrm{U} / \mathrm{ml}$ penicillin, and $100 \mu \mathrm{g} / \mathrm{ml}$ streptomycin at $37{ }^{\circ} \mathrm{C}$ in a humidified $95 \%$ air and $5 \% \mathrm{CO}_{2}$ incubator.

\section{Cell survival assay}

Cell viability was measured using clonogenic and MTT assays. To determine seeding number, different numbers of cells were seeded in 6-well plates without any treatment. For the clonogenic assay, cells were seeded in 6-well plates in triplicate with three different numbers of cells. The cells were fixed after around ten days, stained with $0.1 \%$ crystal violet for ten minutes and counted [27]. Colony formation efficiency is defined as the number of colonies observed/the number of cells plated.

For the MTT assay, HT-29 or HCT-116 cells were seeded in 96-well plates in the presence or absence of eight different concentrations of the treatment chemicals (droxinostat, tubastatin A and PCI-34051) for $48 \mathrm{~h}$. The control cells were supplemented with medium containing DMSO (vehicle control). Following treatment, we performed the MTT assay, which is based on the conversion of MTT to MTT formazan by mitochondrial enzymes.

The effect on cell growth was assessed as a percentage of cell viability, with vehicle-treated cells considered $100 \%$ viable. The $\mathrm{IC}_{50}$ of each chemical was calculated using GraphPad software (GraphPad Software Inc.).

\section{HDAC3 knockdown}

Cultured HT-29 cells in 6-well plates were transfected with: $5 \mathrm{nM}$ HDAC3 on-target plus HDAC3 siRNA-Smart (Ribobio); or 5 nM HDAC3 on-target plus control siRNA using RNAi transfection reagent (Ribobio). The cells were assayed $48 \mathrm{~h}$ post-transfection.

\section{ROS assay and antioxidant treatment}

The levels of ROS were measured using flow cytometry with the probe $2^{\prime}, 7^{\prime}$-dichlorodihydrofluorescein diacetate (DCFDA). Briefly, cells were seeded in 6-well plates, incubated overnight and then treated with $21 \mu \mathrm{M}$ droxinostat for 12 or $24 \mathrm{~h}$. The cells were then detached, centrifuged for $5 \mathrm{~min}$ at $200 \mathrm{x} \mathrm{g}$, and stained in $1 \mathrm{ml}$ of DCFDA staining solution (final concentration of DCFDA is $10 \mu \mathrm{M})$ or MitoSOX Red $(5 \mu \mathrm{M})$ at $37{ }^{\circ} \mathrm{C}$ for $30 \mathrm{~min}$. The stained cells were washed with $3 \mathrm{ml}$ ice-cold phosphate-buffered saline (PBS) and suspended in $200 \mu \mathrm{l}$ of PBS. Dead cells were excluded by PI staining. The ROS level was measured using flow cytometry and presented as the mean fluorescence intensity (MFI) of DCF or MitoSOX Red. 
For antioxidant effects, cells were seeded in 6-well plates, incubated overnight, treated with $10 \mu \mathrm{M}$ GT3 for $2 \mathrm{~h}$, and incubated with $21 \mu \mathrm{M}$ droxinostat for $24 \mathrm{~h}$. The ROS level was also measured via DCFDA using flow cytometry as indicated above.

\section{Apoptotic assay and anti-apoptotic treatment}

Cells seeded in 6-well plates were treated with $21 \mu \mathrm{M}$ of droxinostat for 12 or $24 \mathrm{~h}$. Following incubation, cellular apoptosis was measured using annexin V and PI staining with an FITC Annexin V Apoptosis Detection Kit (BD Biosciences) following the manufacturer's instructions.

For the anti-apoptotic effects, cells were treated with $10 \mu \mathrm{M}$ Z-VAD-FMK at the manufacturer's recommended concentration for $2 \mathrm{~h}$, and then incubated with $21 \mu \mathrm{M}$ of droxinostat for $24 \mathrm{~h}$. Following incubation, cellular apoptosis was assessed by annexin $\mathrm{V}$ and PI staining following the manufacturer's instructions.

\section{Quantitative RT-PCR}

Total RNA was extracted from HT-29 cells using the Qiagen RNeasy Mini Kit according to the manufacturer's instructions. RNA yield and quality were determined by measuring absorbencies at $260 \mathrm{~nm}$ and $280 \mathrm{~nm}$, respectively. First-strand cDNA was synthesized in a final volume of $20 \mu \mathrm{l}$ using the Superscript III First-Strand Synthesis System (Invitrogen). Quantitative RT-PCR analyses were performed using a SYBR Green mix on an ABI StepOne Plus Real-Time PCR System (Applied Biosystems). GAPDH transcripts were used as a housekeeping internal reference for mRNA. The expressions of catalase, superoxide dismutase 1 (SOD1), SOD2 and FLIP-L, Bcl-2, Bcl-xl, BAK, BAX and Puma were calculated using the comparative $C_{T}$ method. The sequences for all the primers used in the quantitative RT-PCR assays are available upon request.

\section{Caspase-3 activity measurement}

The activity of caspase- 3 was measured according to the manufacturer's instructions (Abcam). Briefly, HT-29 cells were suspended in chilled cell lysis buffer after different treatments. Cell lysates were mixed with reaction buffer and DEVD-p-NA substrate for $1 \mathrm{~h}$ at $37{ }^{\circ} \mathrm{C}$. The activity of caspase- 3 was measured on a microplate reader at OD $405 \mathrm{~nm}$ and expressed as the optical density (OD).

\section{H2AX immunostaining}

Cells were fixed with 4\% PFA $6 \mathrm{~h}$ after incubation with droxinostat. After blocking with $1 \%$ bovine serum albumin (BSA), the cells were incubated with anti-phospho-Histone H2AX (1:100, Ser139, Cell Signaling Technology) overnight at $4{ }^{\circ} \mathrm{C}$. After a wash, the cells were stained with Alex 488-conjugated secondary antibody and counterstained with DAPI. The stained cells were viewed and acquired using a Zeiss Axio Observer Z1 microscope (Carl Zeiss Microimaging Inc.) with an Apo 60X/1.4 oil DICIII objective. The images were captured by using AxioVision (4.7.1.0) software (Carl Zeiss). 


\section{Statistical analysis}

The data were analyzed with one-way or two-way ANOVA with SPSS statistic 17.0 (SPSS Software) and GraphPad Prism 5 (GraphPad Software Inc.). $p<0.05$ was considered statistically significant.

\section{Results}

\section{Droxinostat inhibited the growth of human colon cancer cells}

To estimate the effects of droxinostat on the growth of colon cancer cells, 400 HT-29 cells were seeded onto a 96-well plate and treated with different concentrations of droxinostat. Three days later, the MTT assay was used to measure cell numbers in each dose. The data showed that droxinostat treatment inhibited cell growth in a dose-dependent manner starting from $3.125 \mu \mathrm{M}$ (Fig. 1a). Using the nonlinear regression method, the $\mathrm{IC}_{50}$ of droxinostat was about $21 \mu \mathrm{M}$.

To establish the seeded numbers of HT-29 cells for colony assay, we plated 100-4000 cells in 6-well plates. As shown in Additional file 1: Figure S1, the plating efficiencies are $41.00 \%$ per 100 cells, $45.75 \%$ per 200 cells, $54.10 \%$ per 500 cells, $47.30 \%$ per 1000 cells, $43.55 \%$ per 2000 cells and $30.68 \%$ per 4000 cells. We therefore seeded 500 cells in 6-well plates to test the effects of different concentrations of droxinostat on clonogenic ability compared to the clonogenic rate in the control group (56.70\%). A dose of $6.25 \mu \mathrm{M}$ of droxinostat modestly decreased the clonogenic efficiency to $51.90 \%$. However, 12.5 and $25 \mu \mathrm{M}$ of droxinostat significantly decreased the clonogenic rates to 46.20 and $12.07 \%$, respectively (Fig. 1b).

To confirm the inhibition of droxinostat on HDACs, we harvested protein $48 \mathrm{~h}$ after treatment with different concentrations of droxinostat. As shown in Fig. 1c, $10 \mu \mathrm{M}$ of droxinostat efficiently reduced the expression of HDAC3, 6 and 8 compared to treatment with the vehicle alone. Inhibition of HDACs consistently leads to histone

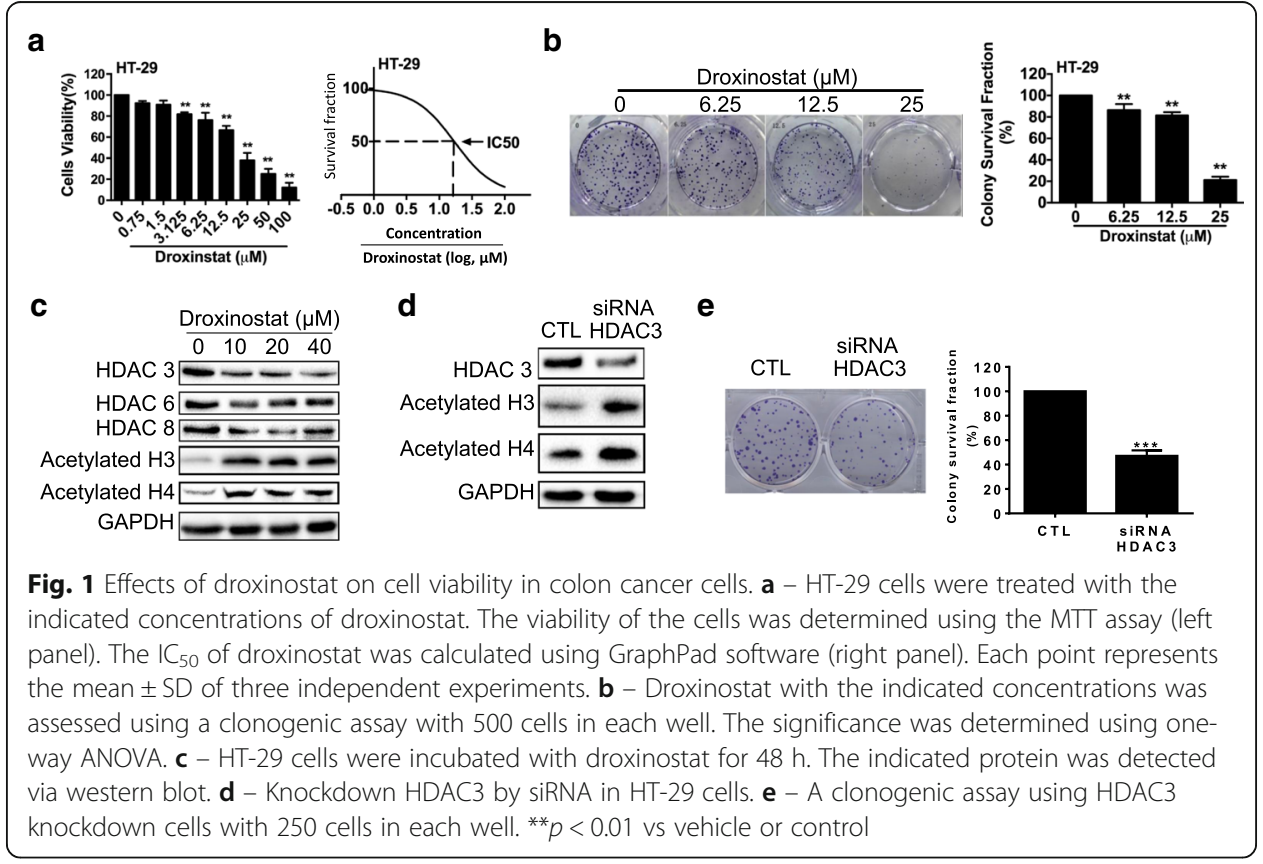


acetylation. Droxinostat treatment obviously increased the expression of acetylated histone $\mathrm{H} 3$ and $\mathrm{H} 4$ compared to vehicle treatment. To mimic the effects of droxinostat on HT-29 cells, we used siRNA to transiently decrease the expression of HDAC3, resulting in increased expression of acetylated histone H3 and H4 (Fig. 1d). The reduction of HDAC3 expression by siRNA significantly decreased the colony-forming ability in HT-29 cells (Fig. 1e). These data indicate that the activity of HDACs plays an important role in colon cancer cell growth.

To investigate whether the negative effect of droxinostat on HT-29 cells is selective among different HDACIs, we tested the effects of tubastatin A and PCI 34051 on HT-29 cells. As shown in Additional file 2: Figure S2A and B, both tubastatin A and PCI 34051 could inhibit cell growth, although the effective concentration of tubastatin A started from $10 \mu \mathrm{M}$ and that of PCI 34051 from $40 \mu \mathrm{M}$. The $\mathrm{IC}_{50}$ values of tubastatin A and PCI 34051 were around 22 and $30 \mu \mathrm{M}$, respectively. Droxinostat, tubastatin A and PCI 34051 not only inhibit the growth of HT-29 cells but also that of HCT-116 colon cancer cells (Additional file 3: Figure S3A-C). We then used droxinostat to further explore the mechanisms by which droxinostat efficiently inhibits cancer cell growth. Our results indicate that HDACIs can inhibit the growth of colon cancer cells.

\section{Droxinostat induced apoptosis in human colon cells}

Previous studies showed that HDACIs can induce cellular apoptosis, resulting in the inhibition of cancer cell growth [8]. In early apoptosis, membrane phosphatidylserine (PS) translocates from the inner face of the cell membrane to the cell surface. Annexin $\mathrm{V}$ can bind to exposed PS with high affinity, whereas PI molecules intercalate inside the DNA double helix in cells with a compromised plasma membrane. We therefore stained cells with annexin V and PI in the absence and presence of droxinostat. As shown in Fig. 2a and b, $21 \mu \mathrm{M}$ of droxinostat induced apoptosis in up to $21.24 \%$ of cells after $12 \mathrm{~h}$ treatment $(p<0.01)$ and in $32.75 \%$ after $24 \mathrm{~h}$ treatment $(p<0.01)$. Notably, the increase in both early and later apoptosis was significant (up to 12.6 and $8.64 \%$ at $12 \mathrm{~h}$, and 23.0 and $9.75 \%$ at $24 \mathrm{~h}$, respectively) with $21 \mu \mathrm{M}$ of droxinstat and 12 and $24 \mathrm{~h}$ treatment $(p<0.01)$, compared to DMSO treated group.

Z-VAD-FMK is an FMK-based pan caspase inhibitor with superior aqueous stability, cell permeability and efficacy but without cytotoxic effects. To further determine the role of cellular apoptosis in the effects of droxinostat on HT-29 cells, we treated cells with $10 \mu \mathrm{M}$ Z-VAD-FMK for $2 \mathrm{~h}$ prior to droxinostat treatment. Fig. 2c shows that Z-VAD-FMK treatment did not increase cellular apoptosis compared to the DMSO group $(p>0.05)$. Z-VAD-FMK pre-treatment could efficiently inhibit the increase in apoptosis induced by droxinostat from 24.12 to $14.26 \%$ with statistical significance $(p<0.01)$. Consistently, droxinostat treatment on HT-29 cells significantly increased the activity of caspase-3, but this effect was reversed by Z-VAD-FMK pre-treatment (Fig. 2d).

Pro- and anti-apoptotic genes play crucial roles during the apoptotic process, so we measured the expressions of apoptosis-related genes with droxinostat and/or Z-VAD-FMK treatment. As shown in Fig. 2e and f, droxinostat treatment increased the expressions of BAK, BAX and Puma while the expressions of Bcl-2 and Bcl-xl decreased with droxinostat treatment. The expression changes of apoptosis-related genes induced by droxinostat were ameliorated by Z-VAD-FMK pre-treatment. These data 


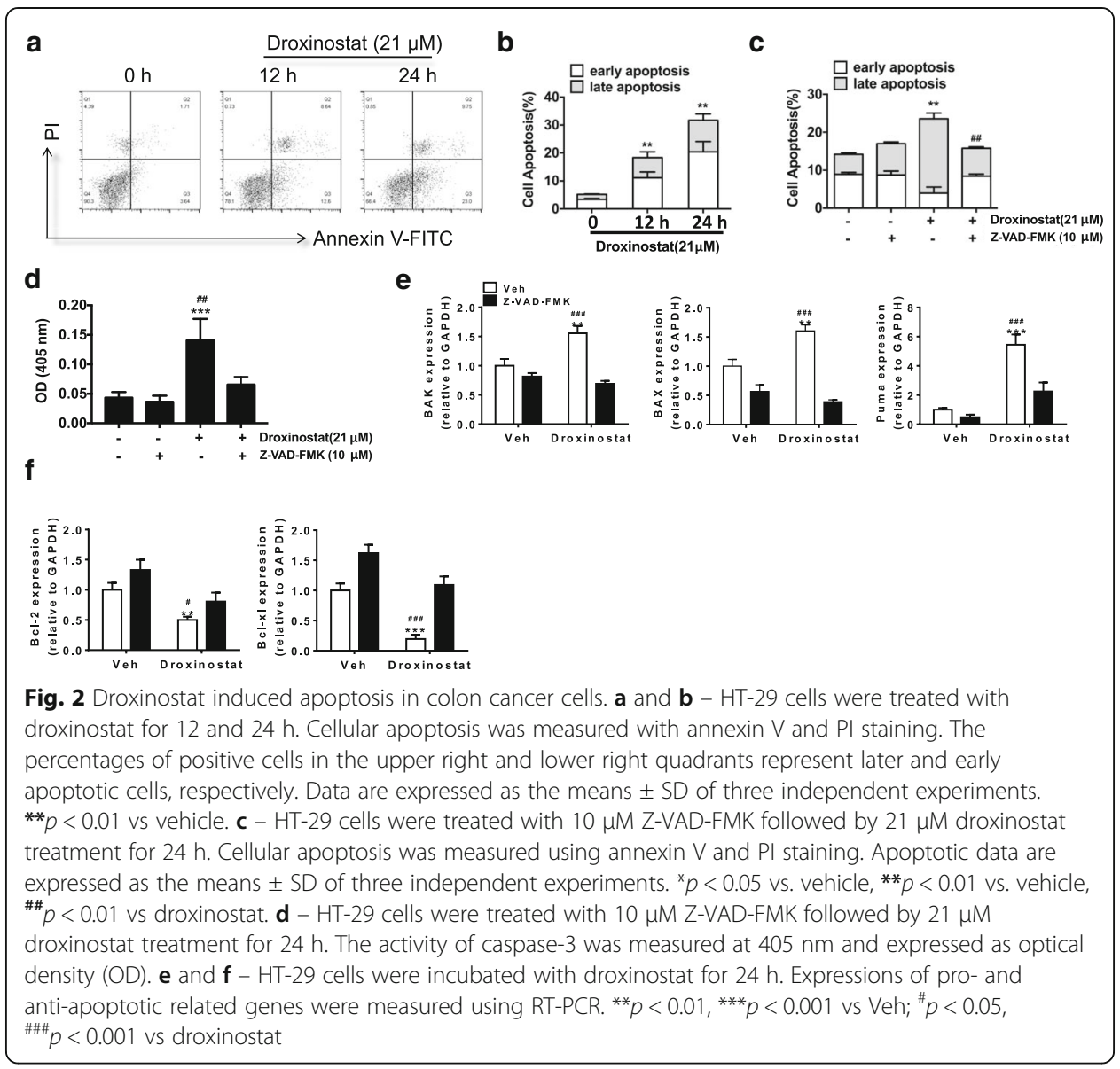

indicated that droxinostat inhibits the growth of cancer cells, and that this is at least in part due to induction of cellular apoptosis.

\section{Droxinostat induces oxidative stress in human colon cells}

HDACIs induce cellular apoptosis and the formation of reactive oxygen species (ROS). To assess whether the induction of ROS plays a role in the inhibition of HT-29 cell growth upon droxinostat treatment, HT-29 cells were incubated with $21 \mu \mathrm{M}$ droxinostat or DMSO. After 12 and $24 \mathrm{~h}$, the production of ROS was measured using a DCFDA probe and then analyzed via flow cytometry.

As shown in Fig. 3a and b, droxinostat significantly increased the accumulation of ROS in cells (around two-fold) $24 \mathrm{~h}$ after treatment $(p<0.01)$. Many antioxidant elements, such as catalase, SOD1 and SOD2, are involved in ROS production. Consistently, droxinostat treatment decreased the expression of catalase, SOD1 and SOD2 when compared to vehicle treatment (Fig. 3c).

MitoSOX Red probe was used to determine whether droxinostat-induced ROS production is derived from the mitochondria. As shown in Fig. 3d, the mean fluorescence intensity of MitoSOX Red was increased (up to 1.5-fold) by droxinostat treatment. These data indicated that droxinostat-induced ROS production is partially derived from the mitochondria. Increased ROS production also causes DNA damage, evidence of 


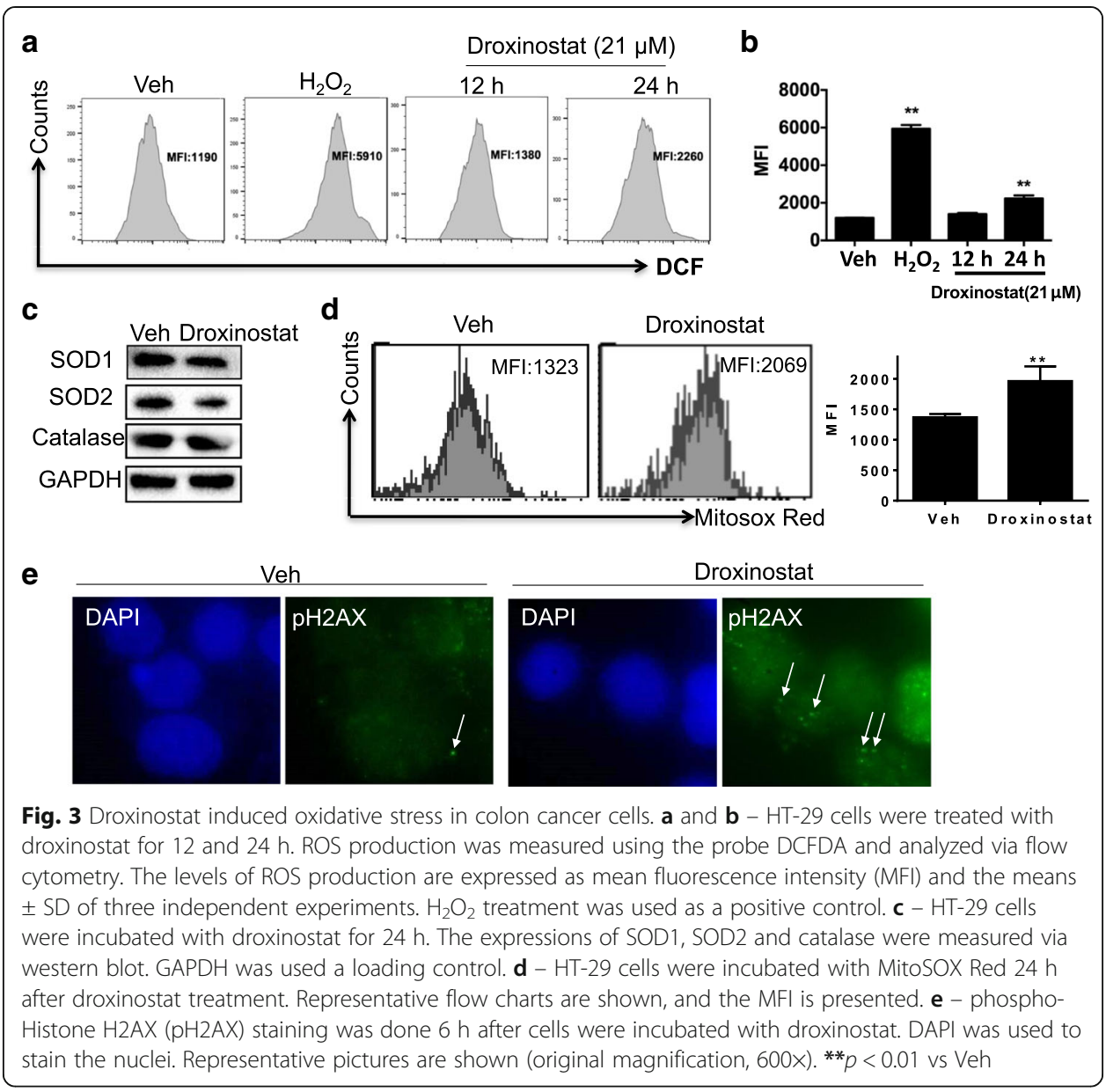

which was obtained with phosphorylated H2AX immunostaining (Fig. 3e). These results suggest that droxinostat induces oxidative stress in human colon cells.

To further confirm the role of ROS production, we pre-treated cells with $10 \mu \mathrm{M}$ GT3 for $2 \mathrm{~h}$ before droxinostat administration. As shown in Fig. 4a, application of GT3 could partially reduce the increased levels of ROS production after droxinostat treatment $(p<0.01)$. The results from the colongenic assay showed that treatment with GT3 reversed the decreased numbers of colonies induced by droxinostat treatment (Fig. 4b). The expression of catalase, SOD1 and SOD2 was significantly decreased by droxinostat treatment (Fig. 4c-e), and this effect was reversed by GT3 pre-treatment. We concluded that induction of ROS might be one of mechanisms in droxinostat-induced inhibition of cancer cell growth.

\section{Oxidative stress induced by droxinostat contributes to cellular apoptosis}

To further explore the interaction between cellular apoptosis and ROS production induced by droxinostat, the ratios of cellular apoptosis were measured after droxinostat and GT3 treatment. As shown in Fig. 5a, GT3 pre-treatment could efficiently decrease droxinostat-induced apoptosis in HT-29 cells. The increased caspase-3 activity and decreased c-FLIP expression with droxinostat treatment were consistently reversed by 


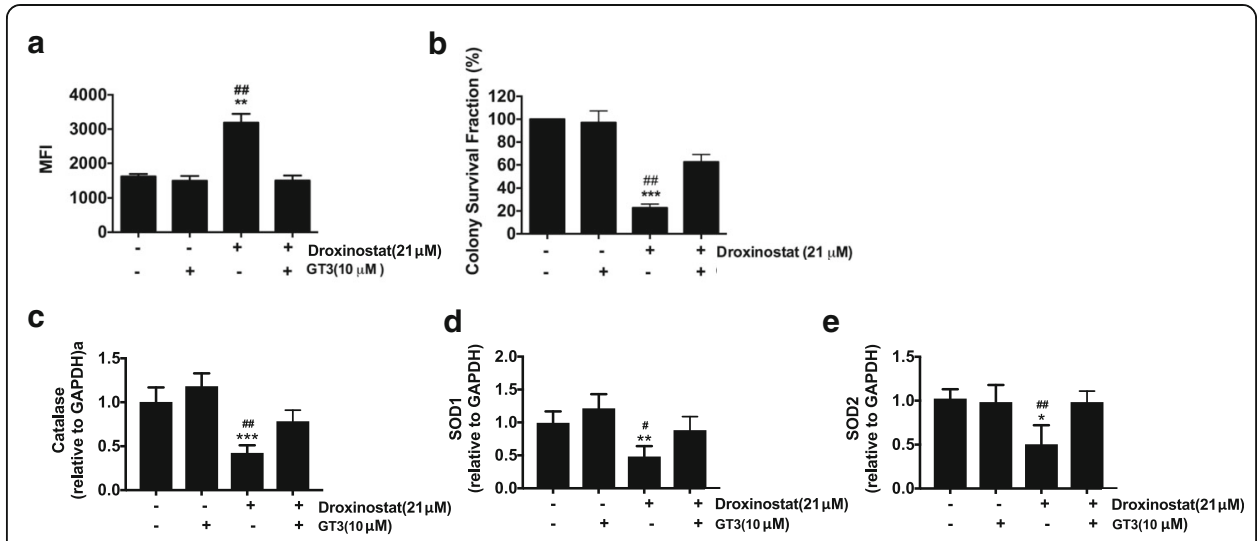

Fig. 4 GT3 pretreatment blocked oxidative stress induced by droxinostat treatment in colon cancer cells. a - HT-29 cells were treated with GT3 followed by droxinostat treatment for $24 \mathrm{~h}$. ROS production was measured using DCFDA and analyzed via flow cytometry. The levels of ROS are expressed as the means \pm SD of three independent experiments. ${ }^{* *} p<0.01$ vs. vehicle, ${ }^{\# \#} p<0.01$ vs. GT3. $\mathbf{b}-500$ HT-29 cells were seeded in 6-well plates and treated with or without GT3 and droxinostat. Ten days later, the colony numbers were counted and are expressed as the colony survival fraction. ${ }^{* *} p<0.001$ vs. vehicle, ${ }^{\# \#} p<0.01$ vs. GT3. C through E - HT-29 cells were treated with GT3 followed by droxinostat treatment for $24 \mathrm{~h}$, and RNA was extracted to measure the expressions of catalase (c), SOD1 (d) and SOD2 (e) via RT-PCR. GADPH was used as a housekeeping control. ${ }^{*} p<0.001$ vs. vehicle, ${ }^{* *} p<0.001$ vs. vehicle, ${ }^{* * *} p<0.001$ vs. vehicle, ${ }^{\#} p<0.05$ vs. GT3, ${ }^{\# \#} p<0.01$ vs. GT3
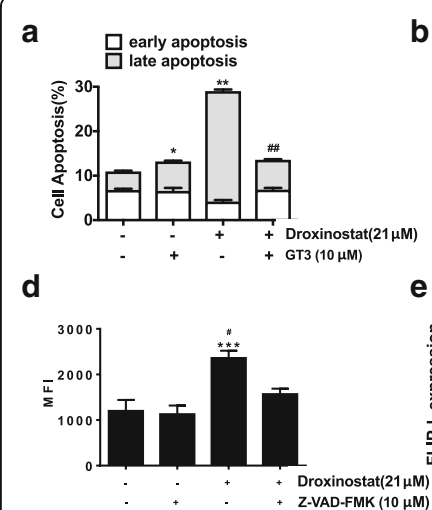

b
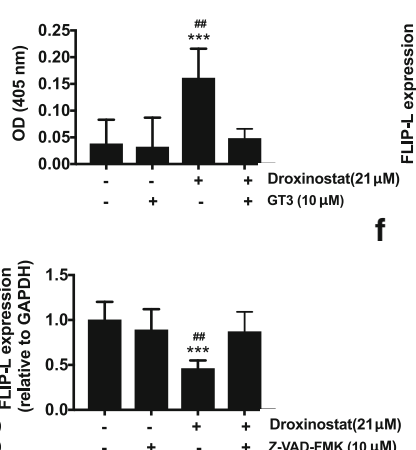

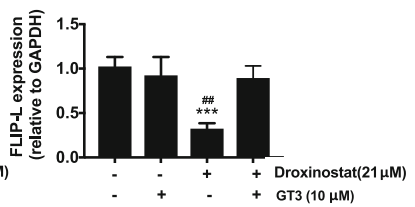

ะ

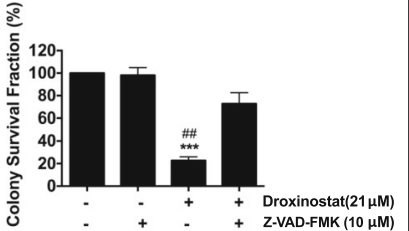

Fig. 5 GT3 pre-treatment decreased droxinostat-induced apoptosis in colon cancer cells. a- HT-29 cells were treated with $10 \mu \mathrm{M}$ GT3 followed by $21 \mu \mathrm{M}$ droxinostat treatment for $24 \mathrm{~h}$. Cellular apoptosis was measured with annexin $V$ and PI staining. Apoptotic data are expressed as the means \pm SD of three independent experiments. ${ }^{*} p<0.05$ vs. vehicle, ${ }^{* *} p<0.01$ vs. vehicle, ${ }^{\# \#} p<0.01$ vs. GT3. $\mathbf{b}-$ HT-29 cells were treated with $10 \mu \mathrm{M}$ GT3 followed by $21 \mu \mathrm{M}$ droxinostat treatment for $24 \mathrm{~h}$. The activity of caspase-3 was measured at $405 \mathrm{~nm}$ and is expressed as optical density (OD). c - HT-29 cells were treated with $10 \mu \mathrm{M}$ GT3 followed by $21 \mu \mathrm{M}$ droxinostat treatment for $24 \mathrm{~h}$. Expression of c-FLIP was assessed via RT-PCR. GADPH was used as a housekeeping control. ${ }^{* * *} p<0.001$ vs. vehicle, ${ }^{\# \#} p<0.01$ vs. GT3. $\mathbf{d}-$ HT-29 cells were treated with $10 \mu \mathrm{M} Z$ Z-VAD-FMK followed by $21 \mu \mathrm{M}$ droxinostat treatment for $24 \mathrm{~h}$. ROS production was measured using DCFDA and analyzed via flow cytometry. The levels of ROS are expressed as the means \pm SD of three independent experiments. ${ }^{* * *} p<0.001$ vs. vehicle, ${ }^{*} p<0.05$ vs. droxinostat. e $-H T-29$ cells were treated with $10 \mu \mathrm{M}$ Z-VAD-FMK followed by $21 \mu \mathrm{M}$ droxinostat treatment for $24 \mathrm{~h}$. Expression of c-FLIP was assessed via RT-PCR. $\mathbf{f}-500 \mathrm{HT}-29$ cells were seeded in 6-well plates and treated with or without Z-VAD-FMK and droxinostat. Ten days later, the colony numbers were counted and are expressed as the colony survival fraction. ${ }^{* *} p<0.001$ vs. vehicle, ${ }^{\# \#} p<0.01$ vs. Z-VAD-FMK 
GT3 treatment (Fig. 5b and c). These findings indicate that droxinostat induces oxidative stress leading to cellular apoptosis.

Z-VAD-FMK pre-treatment decreased droxinostat-induced ROS production, but the production of ROS induced by droxinostat never returned to normal levels (Fig. 5d). A previous study showed that droxinostat decreased the expression of c-FLIP [28, 29]. As shown in Fig. 5e, c-FLIP expression was indeed reduced by droxinostat treatment. Z-VAD-FMK pre-treatment can prevent the decreased expression of c-FLIP with droxinostat treatment.

Lastly, the colongenic assay showed that the killing effects of droxinostat were reversed by Z-VAD-FMK treatment (Fig. 5f). Therefore, oxidative stress and cellular apoptosis affect each other during droxinostat treatment.

\section{Discussion}

The increased expression and activity of histone deacetylase (HDAC) have been documented in different types of cancers [30]. Dysfunctional HDAC result in normal cell transformation and cancer cell resistance to chemotherapeutic drugs [31]. Therefore, inhibition of HDAC activity has potential as a therapeutic approach to limit cancer cell growth. The development of novel effective HDAC inhibitors (HDACIs) is urgent.

Cells can die of apoptosis through the extrinsic death receptor-induced pathway and/ or the intrinsic mitochondrial-mediated pathway [32]. However, programmed cell death is disrupted in cancer, leading to excessive growth of malignant cells [33]. Inducing tumor cell apoptosis is the goal of many cancer therapies.

Our results revealed that droxinostat decreased the expressions of HDAC3, 6 and 8 and increased the expressions of acetylated histone $\mathrm{H} 3$ and H4. Droxinostat treatment increased the activity of capcase-3, leading to cellular apoptosis in HT-29 colon cancer cells. Caspase- 3 plays crucial roles in both the extrinsic death receptor-induced pathway and the intrinsic mitochondrial apoptotic pathway. The consecutive activation of caspase family members is considered the prerequisite of apoptosis [19]. Furthermore, Z-VAD-FMK, an irreversible pan caspase inhibitor, could inhibitor droxinostat-induced apoptosis in HT-29 cells.

The expression of c-FLIP was decreased by droxinostat treatment in HT-29 cells, which resulted in the activation of the extrinsic apoptotic pathway. Waugh et al. and Zhang et al. also found that HDACIs, such as droxinostat and SAHA, downregulated c-FLIP expression in prostatic cancer cells and hepatocellular carcinoma cells [28, 29].

Previous studies and our data from this study show that HDACIs, including droxinostat, induce cellular apoptosis leading to cancer cell death [28, 34, 35]. A recent report showed that the balance disruption of the anti- and pro-oxidant system might be involved in the cancer cell sensitivity of HDACIs [36-38]. For example, inhibition of HDAC5 increased mitochondrial iron-dependent ROS production in HeLa cells, resulting in apoptosis and autophagy [38]. Panobionstat, a pan HDACI, reduced the viability of HeLa cells through induction of ROS [39]. The data from Lassmann et al. also demonstrated that treatment with HDAC inhibitors negatively regulated the expression of thioredoxin with increasing intracellular oxidative stress and cancer cell death [36,37].

Our data showed that droxinostat induced oxidative stress and ROS production in colon cancer cells, which is supported by the decreased expression of catalase, SOD1 and SOD2 with droxinostat treatment. $\gamma$-tocotrienol (GT3) is a vitamin E analogue with 
strong anti-oxidant activity, which can decrease the high levels of oxidative stress. Our results indicated that GT3 pre-treatment restored the colony-forming ability affected by droxinostat treatment. This is because GT3 reduces the increased ROS production induced by droxinostat and increases the expression of catalase, SOD1 and SOD2. Notably, GT3 pre-treatment decreased cellular apoptosis, increased c-FLIP expression and reduced the increased caspase-3 activity seen with droxinostat treatment.

Our findings suggest that the increase of ROS production induced by droxinostat in colon cancer cells might further result in apoptotic cell death. Similarly, inhibition of droxinostat-induced apoptosis can partially decrease ROS production under droxinostat treatment.

In summary, we demonstrated that droxinostat effectively reduces the viability of colon cancer cells, and that this is mediated by the induction of oxidative stress and cellular apoptosis. Therefore, droxinostat might have potential as a novel therapeutic agent for the treatment of colon cancer.

\title{
Additional files
}

Additional file 1: Figure S1 Colony-forming assay. 100-4000 HT-29 cells were seeded in 6-well plates. The cell culture medium was changed every two days. The colonies were counted ten days after plating (A). Plating efficiency (\%) was calculated as the number of colonies observed/the number of cells plated (B). (PPTX 179 kb)

Additional file 2: Figure S2 Effects of tubastatin and PCl-34051 of cell viability in HT-29 colon cancer cells. HT-29 cells were treated with the indicated concentrations of tubastatin A (A) and PCl-34051 (B). The viability of the cells was determined using the MTT assay. Each point represents the mean \pm SD of three independent experiments. The significance was determined using the one-way ANOVA. ${ }^{*} p<0.05$ vs. vehicle, ${ }^{* *} p<0.01$ vs. vehicle. (PPTX $\left.841 \mathrm{~kb}\right)$

Additional file 3: Figure S3 Effects of droxinostat, tubastatin and PCl-34051 of cell viability in HCT-116 colon cancer cells. HCT-116 cells were treated with the indicated concentrations of droxinostat (A), tubastatin $A(B)$ and PCl34051 (C). The viability of the cells was determined using the MTT assay. Each point represents the mean \pm SD of three independent experiments. The significance was determined using the one-way ANOVA. * $p<0.05$ vs. vehicle. (PPTX 76 kb)

\begin{abstract}
Abbreviations
5-FU: 5-fluorouracil; ANOVA: Analysis of variance; c-FLIP: Cellular FLICE (FADD-like IL-1ß-converting enzyme)-inhibitory protein; CRC: Colorectal cancer; DAPI: 4',6-diamidino-2-phenylindole; DCFDA: 2',7'-dichlorodihydrofluorescein diacetate; DISC: Death-inducing signaling complex; GT3: $\gamma$-tocotrienol; HATs: Histone acetyltransferases; HDACls: Histone deacetylase inhibitors; HDACs: Histone deacetylases; MFI: Mean fluorescence intensity; MTT: 3-(4, 5-dimethylthiazolyl-2)2, 5-diphenyltetrazolium bromide; PI: Propidium iodide; PS: Phosphatidylserine; ROS: Reactive oxygen species; SAHA: Vorinostat; siRNA: Small interfering RNA; SOD1: Superoxide dismutase 1; TRAIL: Tumor necrosis factor-related apoptosis-inducing ligand
\end{abstract}

Acknowledgements

The authors would like to thank Mr. Huixing Deng for the English language review.

Funding

This study was financially supported by grant from the National Natural Science Foundation of China (No. 81160050, 81460110).

Availability of data and materials

All data generated or analyzed during this study were included in this published article and its supplementary information files.

Authors' contributions

$\mathrm{LS}$ and BK conceived and designed the experiments. $\mathrm{YH}, \mathrm{HZ}, \mathrm{CH}$ and $\mathrm{YZ}$ performed the experiments. ND and GF helped to analyze the data. LS and BK wrote the paper. All authors read and approved the final manuscript.

Ethics approval and consent to participate

The data presented in this manuscript were generated from in vitro cell culture. Human or animal samples were not used. 


\section{Competing interests}

The authors declare that they have no competing interests.

\section{Publisher's Note}

Springer Nature remains neutral with regard to jurisdictional claims in published maps and institutional affiliations.

\section{Author details}

${ }^{1}$ Jiangxi provincial key laboratory of preventive medicine, Nanchang University, Nanchang 330006, China. ${ }^{2}$ Medical School of Nanchang University, 461 Bayi Road, Nanchang 330006, Jiangxi, China. ${ }^{3}$ School of Public Health, Nanchang University, Nanchang 330006, China.

Received: 4 January 2018 Accepted: 19 July 2018

Published online: 28 July 2018

\section{References}

1. Weitz J, Koch M, Debus J, Hohler T, Galle PR, Buchler MW. Colorectal cancer. Lancet. 2005;365:153-65.

2. Favoriti P, Carbone G, Greco M, Pirozzi F, Pirozzi RE, Corcione F. Worldwide burden of colorectal cancer: a review. Updat Surg. 2016:68:7-11.

3. McLornan DP, Barrett HL, Cummins R, McDermott U, McDowell C, Conlon SJ, et al. Prognostic significance of TRAIL signaling molecules in stage II and III colorectal cancer. Clin Cancer Res. 2010;16:3442-51.

4. Stevenson L, Allen WL, Turkington R, Jithesh PV, Proutski I, Stewart G, et al. Identification of galanin and its receptor GalR1 as novel determinants of resistance to chemotherapy and potential biomarkers in colorectal cancer. Clin Cancer Res. 2012;18:5412-26.

5. Galligan L, Longley DB, McEwan M, Wilson TR, McLaughlin K, Johnston PG. Chemotherapy and TRAIL-mediated colon cancer cell death: the roles of p53, TRAIL receptors, and c-FLIP. Mol Cancer Ther. 2005;4:2026-36.

6. Pontiki E, Hadjipavlou-Litina D. Histone deacetylase inhibitors (HDACls). Structure--activity relationships: history and new QSAR perspectives. Eur J Med Chem. 2012;32:1-165.

7. Monneret C. Histone deacetylase inhibitors. Eur J Med Chem. 2005;40:1-13.

8. Han MH, Park C, Kwon TK, Kim GY, Kim WJ, Hong SH, et al. The Histone Deacetylase Inhibitor Trichostatin A Sensitizes Human Renal Carcinoma Cells to TRAlL-Induced Apoptosis through Down-Regulation of c-FLIPL. Biomo Ther (Seoul). 2015:23:31-8.

9. Rao-Bindal K, Koshkina NV, Stewart J, Kleinerman ES. The histone deacetylase inhibitor, MS-275 (entinostat), downregulates C-FLIP, sensitizes osteosarcoma cells to FasL, and induces the regression of osteosarcoma lung metastases. Curr Cancer Drug Targets. 2013;13:411-22.

10. Zheng Z, Cheng S, Wu W, Wang L, Zhao Y, Shen Y, et al. c-FLIP is involved in tumor progression of peripheral Tcell lymphoma and targeted by histone deacetylase inhibitors. J Hematol Oncol. 2014;7:88.

11. Kauh J, Fan S, Xia M, Yue P, Yang L, Khuri FR, et al. C-FLIP degradation mediates sensitization of pancreatic cancer cells to TRAIL-induced apoptosis by the histone deacetylase inhibitor LBH589. PloS one. 2010;5:e10376.

12. Hengartner MO. The biochemistry of apoptosis. Nature. 2000;407:770-6.

13. Elrod HA, Lin YD, Yue P, Wang X, Lonial S, Khuri FR, et al. The alkylphospholipid perifosine induces apoptosis of human lung cancer cells requiring inhibition of Akt and activation of the extrinsic apoptotic pathway. Mol Cancer Ther. 2007:6:2029-38.

14. Wilson TR, Redmond KM, McLaughlin KM, Crawford N, Gately K, O'Byrne K, et al. Procaspase 8 overexpression in non-small-cell lung cancer promotes apoptosis induced by FLIP silencing. Cell Death Differ. 2009;16:1352-61.

15. Rao-Bindal K, Zhou Z, Kleinerman ES. MS-275 sensitizes osteosarcoma cells to Fas ligand-induced cell death by increasing the localization of Fas in membrane lipid rafts. Cell Death Dis. 2012;3:e369.

16. Zhao L, Yue P, Khuri FR, Sun SY. mTOR complex 2 is involved in regulation of Cbl-dependent c-FLIP degradation and sensitivity of TRAlL-induced apoptosis. Cancer Res. 2013;73:1946-57.

17. Lavrik I, Golks A, Krammer PH. Death receptor signaling. J Cell Sci. 2005;118:265-7.

18. Liu X, Yue P, Chen S, Hu L, Lonial S, Khuri FR, et al. The proteasome inhibitor PS-341 (bortezomib) up-regulates DR5 expression leading to induction of apoptosis and enhancement of TRAlL-induced apoptosis despite upregulation of C-FLIP and survivin expression in human NSCLC cells. Cancer Res. 2007;67:4981-8.

19. Zhao L, Wen Q, Yang G, Huang Z, Shen T, Li H, et al. Apoptosis induction of dehydrobruceine B on two kinds of human lung cancer cell lines through mitochondrial-dependent pathway. Phytomedicine. 2016;23:114-22.

20. Schimmer AD, Thomas MP, Hurren R, Gronda M, Pellecchia M, Pond GR, et al. Identification of small molecules that sensitize resistant tumor cells to tumor necrosis factor-family death receptors. Cancer Res. 2006;66:2367-75.

21. Mawji IA, Simpson CD, Hurren R, Gronda M, Williams MA, Filmus J, et al. Critical role for Fas-associated death domain-like interleukin-1-converting enzyme-like inhibitory protein in anoikis resistance and distant tumor formation. J Natl Cancer Inst. 2007;99:811-22.

22. Wood TE, Dalili S, Simpson CD, Sukhai MA, Hurren R, Anyiwe K, et al. Selective inhibition of histone deacetylases sensitizes malignant cells to death receptor ligands. Mol Cancer Ther. 2010;9:246-56.

23. Kerr E, Holohan C, McLaughlin KM, Majkut J, Dolan S, Redmond K, et al. Identification of an acetylation-dependant Ku70/FLIP complex that regulates FLIP expression and HDAC inhibitor-induced apoptosis. Cell Death Differ. 2012; 19:1317-27.

24. Lin Y, Liu X, Yue P, Benbrook DM, Berlin KD, Khuri FR, et al. Involvement of C-FLIP and survivin down-regulation in flexible heteroarotinoid-induced apoptosis and enhancement of TRAlL-initiated apoptosis in lung cancer cells. Mol Cancer Ther. 2008;7:3556-65.

25. Sun S, Han Y, Liu J, Fang Y, Tian Y, Zhou J, et al. Trichostatin a targets the mitochondrial respiratory chain, increasing mitochondrial reactive oxygen species production to trigger apoptosis in human breast cancer cells. PLoS One. 2014;9:e91610. 
26. Ivanova D, Zhelev Z, Aoki I, Bakalova R, Higashi T. Overproduction of reactive oxygen species - obligatory or not for induction of apoptosis by anticancer drugs. Chin J Cancer Res. 2016;28:383-96.

27. Buch K, Peters T, Nawroth T, Sanger M, Schmidberger H, Langguth P. Determination of cell survival after irradiation via clonogenic assay versus multiple MTT assay--a comparative study. Radiat Oncol. 2012;7:1.

28. McCourt C, Maxwell P, Mazzucchelli R, Montironi R, Scarpelli M, Salto-Tellez M, et al. Elevation of c-FLIP in castrateresistant prostate cancer antagonizes therapeutic response to androgen receptor-targeted therapy. Clin Cancer Res. 2012;18:3822-33.

29. Liu J, Li G, Wang X, Wang L, Zhao R, Wang J, et al. Droxinostat, a histone Deacetylase inhibitor, induces apoptosis in hepatocellular carcinoma cell lines via activation of the mitochondrial pathway and Downregulation of FLIP. Transl Oncol. 2016;9:70-8.

30. Damaskos C, Garmpis N, Valsami S, Kontos M, Spartalis E, Kalampokas T, et al. Histone Deacetylase inhibitors: an attractive therapeutic strategy against breast Cancer. Anticancer Res. 2017;37(1):35-46.

31. Zahnow CA, Topper M, Stone M, Murray-Stewart T, Li H, Baylin S, et al. Inhibitors of DNA methylation, histone Deacetylation, and histone Demethylation: a perfect combination for Cancer therapy. Adv Cancer Res. 2016;130: 55-111.

32. Zimmermann KC, Green DR. How cells die: apoptosis pathways. J Allergy Clin Immunol. 2001;108:S99-103.

33. Li H, Li C, Shen T, Zhao L, Ren D. R-eriodictyol and S-eriodictyol exhibited comparable effect against $\mathrm{H}_{2} \mathrm{O} 2-$ induced oxidative stress in EA.hy926 cells. Drug Discov Ther. 2014;8:218-24.

34. Anantharaju PG, Reddy DB, Padukudru MA, Chitturi CMK, Vimalambike MG, Madhunapantula SV. Induction of colon and cervical cancer cell death by cinnamic acid derivatives is mediated through the inhibition of histone Deacetylases (HDAC). PLoS One. 2017;12:e0186208.

35. Shi XY, Ding W, Li TQ, Zhang YX, Zhao SC. Histone Deacetylase (HDAC) inhibitor, Suberoylanilide Hydroxamic acid (SAHA), induces apoptosis in prostate Cancer cell lines via the Akt/FOXO3a signaling pathway. Med Sci Monit. 2017;23:5793-802.

36. Ungerstedt JS, Sowa Y, Xu WS, Shao Y, Dokmanovic M, Perez G, et al. Role of thioredoxin in the response of normal and transformed cells to histone deacetylase inhibitors. Proc Natl Acad Sci U S A. 2005;102:673-8.

37. Ahrens TD, Timme S, Ostendorp J, Bogatyreva L, Hoeppner J, Hopt UT, et al. Response of esophageal cancer cells to epigenetic inhibitors is mediated via altered thioredoxin activity. Lab Investig. 2016;96:307-16.

38. Hendrick E, Peixoto P, Blomme A, Polese C, Matheus N, Cimino J, et al. Metabolic inhibitors accentuate the antitumoral effect of HDAC5 inhibition. Oncogene. 2017;36:4859-74.

39. Wasim L, Chopra M. Panobinostat induces apoptosis via production of reactive oxygen species and synergizes with topoisomerase inhibitors in cervical cancer cells. Biomed Pharmacother. 2016;84:1393-405.

Ready to submit your research? Choose BMC and benefit from:

- fast, convenient online submission

- thorough peer review by experienced researchers in your field

- rapid publication on acceptance

- support for research data, including large and complex data types

- gold Open Access which fosters wider collaboration and increased citations

- maximum visibility for your research: over $100 \mathrm{M}$ website views per year

At $\mathrm{BMC}$, research is always in progress.

Learn more biomedcentral.com/submissions 